\title{
Fabric-circle-slider: Prototype Exploring the Interaction Aesthetic of Contextual Integration
}

\author{
John Zimmerman ${ }^{1,2}$, Amy K. Hurst ${ }^{1}$, \\ Michel M. R. Peeters ${ }^{3}$ \\ ${ }^{1}$ Human-Computer Interaction Institute \\ ${ }^{2}$ School of Design \\ Carnegie Mellon University \\ \{johnz, akhurst\} @andrew. cmu.edu \\ ${ }^{3}$ Department of Industrial Design \\ Technisch Universiteit Eindhoven \\ M.M.R.Peeters@student.tue.nl
}

\begin{abstract}
Traditionally designers have explored the aesthetics of interaction through the relationship between the product form and the activity people use it for. However, in the increasing complexity of interconnected and multi-activity devices in the home, aesthetics have been sacrificed in a move to increase usability. In this paper we present an emerging theory that interaction designs that take a contextual integration approach can draw interaction aesthetics from the context instead of the activity in order to address the increased complexity. In addition, we present a conceptual interaction widget, called the fabric-circleslider that draws its interaction aesthetic from a lounge chair-the context of use-and supports interaction with many devices.
\end{abstract}

\section{Introduction}

Traditionally, simple interactive devices derived their interaction aesthetics from the intersecting relationships between product form, the users' body, and the activity the product supports. The corded telephone offers a beautiful example. The handle shape of the receiver offers a strong perceptual-affordance for grasping. The form and interaction allow users to gracefully express their intent to both receive a phone call by grasping and lifting the receiver and to terminate a call by placing the receiver in the cradle. This approach to interaction works well for simple devices and allows great harmony between the product form and the interaction design.

Today, the rapid growth in both number and complexity of devices in the home has made even simple tasks increasingly difficult. The problem stems from both an increase in the number of devices needed for a single activity and the number of activities a single device supports. Interacting with a TV offers a good example of the more-devices-per-activity phenomenon. Today, users often need to interact with three or four devices just to watch TV. They need to turn on their TV; turn on their cable, satellite or digital video recorder settop box; turn on their sound system; and set all of 
these devices to their appropriate input and output settings. DVD players offer a good example of multiple activities in one device. While originally designed for watching pre-recorded content such as movies, many DVD players currently support recording of TV shows, file transfers between DVDs and hard drives, and audio playback of CDs. The increase in devices per activity and in activities per device has disrupted the intersecting relationships between form, body, and activity. This disruption along with increased interest and investment in usability has lead to a dramatic reduction of interaction aesthetic in current products.

Interaction designers construct their designs by combining different interaction widgets. This atomic currency of an interface contains two parts. First, interaction widgets need a method for the user to express their intent. Second, the systems these widgets communicate with require a specific input type in order to respond appropriately to the user's intent. For example, consider a volume slider and a car accelerator as two interaction widgets. They each provide very different methods for users to express their intent; however, from the system input perspective they are the same, both requiring a single value from an analog, linear list of possible values. Designers construct interfaces by arranging a series of interaction widgets through a consideration of user needs and mental model of the task, the application and activity the device supports, and the physical, environmental, cultural, and social constraints found in the context of use. Traditionally the aesthetics of interaction have drawn heavily from the specific activity a device supports, and they have been diminished by the increasing complexity.

We feel one method for bringing the consideration of interaction aesthetics back into focus in this more complex world is through contextual integration: linking the interaction aesthetics to the elements and resources found in the context. This approach allows a small, extensible set of interaction widgets to control the larger number of devices in the home and can more easily address inter-device connectivity by moving the interaction into the resources and elements in the context and away from the individual devices. To further explore and evaluate this emerging theory we have developed the fabric-circle-slider, which allows users to control different devices from a lounge chair situated in the social environment of a living room. Instead of designing widgets specific to any activity or device--such as pushing buttons on a remote to adjust the volume while watching TV or turning on a lamp in order to read, the fabric-circle-slider builds on the user's physical relationship with their lounge chair. It provides a generic input platform that can be easily extended to control relevant devices found in the living room, allowing users to extend their interaction knowledge across the many different activities they perform in a specific context. For more details on contextual integration and mode design examples, please see [13].

\section{Related Work}

Many design researchers have explored the aesthetics of interaction, producing new directions for exploration, new design methods, frameworks for exploring aesthetic in interaction, and of course many beautiful and inspiring artifacts. One of the main 
interaction themes to come from this work has been a movement toward "tangible" interaction: allowing users to interact with detached physical objects instead of pushing buttons on an individual device. Hiroshi Ishii and his tangible group at MIT have particularly championed this movement away from the screen toward physical objects [8]. One of the early and most inspiring concepts to emerge from the exploration of tangible interaction and aesthetics is Durrel Bishop's telephone answering machine [1]. This interactive device uses marbles as a physical representation of individual messages. The elegant solution of making the intangible message a physical object increases the aesthetic value of the interaction while at the same time supporting the usability of the answering machine. This work relates, informs and inspires our theory of contextual integration in that the method of interaction is not strongly tied to the activity of managing messages and can easily be extended to many different devices that allow users to select an item from a short list.

In addition to the tangible movement, design researchers, particularly in the Netherlands, have been developing both design methods for exploring interaction aesthetics [3] and frameworks for emotionally expressive interactions that leverage both the perceptive and expressive abilities of the human body [11, 12]. Our work is motivated by Djajadiningrat et al.'s interaction relabeling method for exploring interaction aesthetic in that we want to transition the interaction from the electronic device to the resources and elements found in the context, and our process of moving device control to the arm chair is certainly a form of relabeling [3]. We deviate from their method in that we use the relabeling not to inspire how interaction might be done, but as an actual platform for device control. Our work relates to Wensveen et al.'s exploration of emotionally rich interaction in that we try to match the interaction to the user intent and not to a specific device, task, or activity [11, 12]; however, in our case we are tying the interaction explicitly to device control instead of using it as a method for more abstract communication.

Finally, our work has been particularly inspired by two interaction designs with strong elements of contextual integration that increase their interaction aesthetic. The first is Hiroshi Ishii's bottle interface [9]. Users interact with this system by removing glass stoppers and allowing music to flow (turn on) and replacing the stopper to stop the music from playing. We find the design's leveraging of people's interaction experience with bottles to be a nice example of contextual integration in that the bottles relate much more to the context than to the user's connection to music and the activities that surround listening to music. Bill Gaver et al.'s drift table is the other design that particularly inspires our theory [6]. Users interact with the table by placing resources and elements found in the context, such as a glass of beer, on to the table. The table then interprets the change in weight and reacts. This design offers a great example of contextual integration in that the interaction derives naturally from the way people currently use tables. However, this design deviates from our emerging theory in that the interactions the user makes more closely relate to the specific activity of drifting across the countryside, and do not generalize to the other activities they may engage in around the same table. 


\section{Design Process}

The design of the fabric-circle-slider grew out of a larger collaboration we had with the Project on People and Robots [10] to develop assistive products for elders. During previous ethnographic research the Project on People and Robots performed with elders, the chair-side was identified as an opportunity area for assistive products [4]. As elders begin to experience decline in their physical and/or cognitive abilities, their physical world begins to constrict. They begin to spend more of their time at home and conduct more of their daily activities from their favorite chair. To extend this work, we performed a second round of ethnographic research focused more specifically on elders" "favorite chairs". We noted all of the materials and controls they surround themselves with including remote controls, kitchen timers, telephones, and lamps within arm's reach [7]. In addition, we conducted directed storytelling interviews to explore both how they used and how they felt about their chair. Following these interviews we generated a large set of assistive product concepts and conducted a concept validation session with a different set of elders. During this session we explored the idea of a "command chair" that would allow elders to take control of different devices in the home. The elders participating in this session seemed quite interested in this concept and felt it could greatly impact their lives. For more details on the design of the smart lounge chair, please see [5].

Based on the results of the ethnographies and the concept validation session, we selected a project focus of designing an interaction widget for the lounge chair that allowed users to control devices such as their TV, a timer, and lights. In addition, we wanted this work to explore our emerging idea on contextual integration: a design that draws its interaction aesthetic from the context, in this case the lounge chair situated in a living room, instead of the specific devices and activities the widget allows users to control. In taking this approach, we identified two sub-themes that built on the idea of contextual integration:

- Chair-ness: We began using the term "chair-ness" to help ourselves focus on an interaction aesthetic drawn from the chair. In addition, this term/theme came to mean a desire for a pleasurable interaction that matched the comfort and self-soothing support that the favorite lounge chair provides as well as the utility and usability important for successful device control.

- Extensibility: While the design of the smart lounge chair focused on elders, we felt that control of devices from a lounge chair would be valuable to a much larger audience. So while elders remained a focus, we kept a larger audience of home lounge chair users in mind. Also, while we wanted the device to control objects that we found common in our user studies (a timer, the TV, and lights) we wanted to construct a generic widget that could easily be extended to control a variety of devices that this larger audience might want to control from their chair.

\subsection{Design Process}

As a first step in exploring "chair-ness" interaction, we reviewed videotapes made during the ethnographies of elders using their chairs to better understand their natural 
behavior and unconscious interaction dialogues in and with a chair. We also videotaped ourselves sitting in a Lazy Boy chair and performing tasks such as watching $\mathrm{TV}$, reading, sleeping, stretching, sitting down, standing up, etc. Through this exploration we found several interaction sweet-spots: locations on the chair that offer the best opportunity for interaction as well as locations that are already heavily used. Not surprisingly, these spots often reveal themselves on a chair, appearing as faded and balding spots on the top-front of armrests, front of seat, and top of back of the chair (Fig 1). We also identified secondary interaction spots such as the front, inside and outside of the armrests. While a little more difficult to reach, these locations had the advantage of reducing unintentional communication with the chair during people's normal activities.

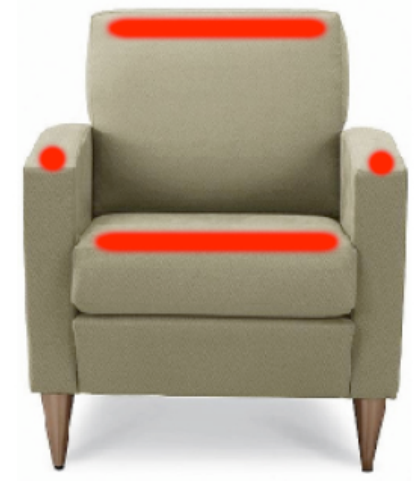

Fig 1. Interaction "sweet spots" on the lounge chair.

Following the exploration of the chair as an interaction platform, we generated many new interaction widgets for users to express their intent to the chair. After an iterative process of sketching and brainstorming different concepts, we selected 12 of the concepts based on their extensibility, appropriate mapping to chair sweet-spots, interaction breadth (extensibility), and on our ability create from a technological standpoint. We then mocked up these concepts as foam core prototypes (Fig 2).

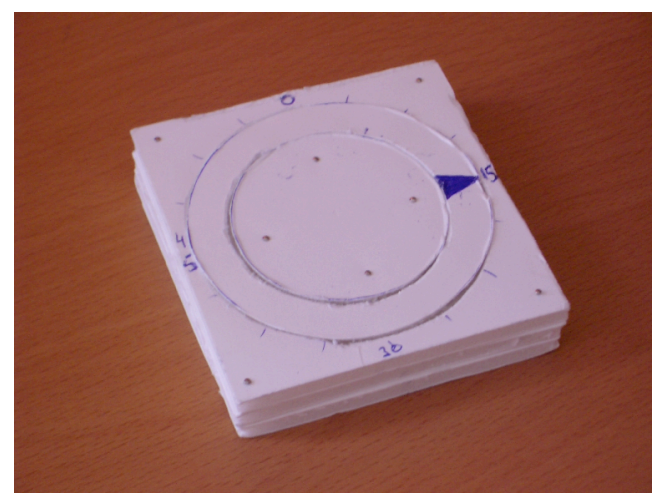


Fig 2. Foam core prototype of a circular slider

In order to evaluate these designs, we performed a think-aloud evaluation with five participants. Our goal was to evaluate both the usability as well as the "chair-ness". During the evaluation sessions we had the participants sit in a lounge chair and interact with the different foam core models, pretending to control a timer, TV volume, and TV channel. In addition to telling us how they thought the widgets worked, participants spoke freely about how they wanted the controls to work and where on the chair they thought they should be located. After completing the think-aloud, we asked participants to rate the different methods using a series of Lickert scale that addressed the intention-interaction appropriateness and the "chair-ness" of the different designs.

In reviewing both the participants' ratings and their comments on the different widgets, it appeared clear that the circular inputs (similar to Apple's iPod) worked very well for expressing a value within a linear list. Additionally, when the circular input was placed on the right-armrest of the chair and was soft, like the padded fabric of the chair, participants found the interaction to be very "chair-ish". The evaluation also revealed two design challenges. First, the placement of an interaction widget on the top-front of the armrest presents a problem with accidental interaction as users engage this location to assist themselves in sitting down, getting up, and other activities they perform in the chair. Second, the lack of feedback from the slider may make it a difficult control to use with devices that do not offer their own form of feedback.

\section{Fabric-Circle-Slider}

Based on the needs of the elder users, our emerging theory of contextual integration with respect to the lounge chair, and the insights from the brief evaluation of the different widgets, we generated the design of the fabric-circle-slider. The widget consists of a sunken ring that surrounds a raised center button, and the widget itself has been integrated into the right, removable armrest cover of a lounge chair (Fig 3). In addition, the fabric-circle-slider has four quasi-mode soft-buttons integrated into the inside flap of the left, removable armrest cover. Users target the device they want to control by pressing and holding (like the shift key found on most keyboards) the appropriate quasi-mode button. Then, with their right hand they can circle their finger around the sunken-ring or press the center button. 


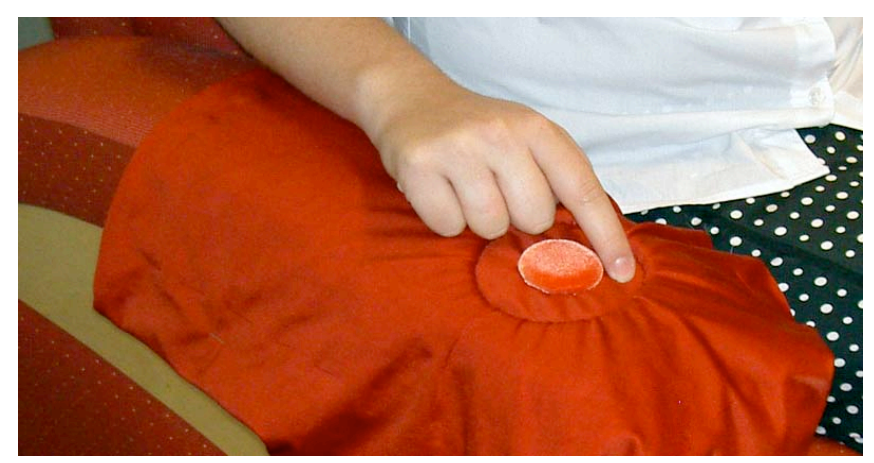

Fig 3. Current prototype of fabric-circle-slider in use.

The use of the quasi-mode buttons offers two advantages. First, it allows users to control several devices from the chair. Second, since users must actively press these buttons to activate the ring-slider, the likelihood of unintentional activation getting in or out of the chair is reduced. Unfortunately scalability is a problem with this quasi mode approach. While the inside flap of the armrest might accommodate many buttons, it is unlikely that users would be able to remember the mapping of the buttons to their device and successfully select one of many buttons without looking at them. Our solution is to limit the design to four buttons, where three control the most commonly used devices and one displays a list of less frequently used devices on the television for users to select.

The ring on the fabric widget supports three interaction expressions: Jog, Shuttle, and Step-In (Fig 4).

- Jog: This expression works like the jog controller found on many high-end VCRs. Users interact by stroking their finger around the ring, expressing a desire to move backwards or forwards within a list. Like the jog controller on a VCR, the interaction is intended to offer users detailed control of their selection. Unlike the jog controller on a VCR, ours employs a speed-ramp, allowing users to increasing and decrease the size of each step they move backwards and forwards in a list by increasing or decreasing the speed of their finger.

- Shuttle: This expression works like a shuttle controller found on many highend VCRs and allows users to set and maintain a specific rate of speed for moving backwards and forwards in a list.

- Step-In: This expression allows users to roughly target a location within a list in order to start a shuttle or jog interaction. The navigation of an alphabetically sorted list provides a good example. Users can use the step-in expression to get very close to the letter they want. They can then use the jog or shuttle expression to move backwards or forwards to the specific item they are looking for. 

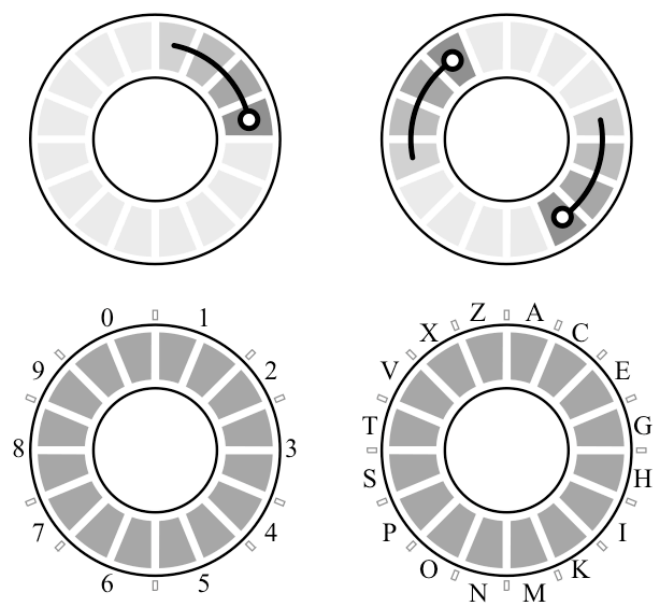

Fig 4. Interactive expressions with fabric-circle-slider (Clockwise starting at top left): 1. Jog, 2. Shuttle, 3. Step-In for alphabetical list, and 4. Step-In for numeric list.

The shuttle and jog expressions can be implemented to either work together or to control separate features of the same device. Using TV control as an example, when combined, users can use the shuttle expression to rapidly scan through channels, quickly jumping from channel 2 to channel 335. When users near the channel they want, they can remove one finger from the ring, transitioning to the jog expression, and more easily select the specific channel they want. In the case of controlling different elements of the same device, using the same TV control example, users could use the shuttle expression to dial the channel they want and use the jog expression for control of the volume. The advantage of this approach is that it eliminates the need for two different quasi-mode buttons; however, remembering which expression does what across many devices would increase the user's cognitive load.

\subsection{Hardware Design}

The fabric-circle-slider employs a set of binary switches made out silk organza, a flexible conductive fabric. Individual sensors get "triggered" when one piece of the silk organza with a positive charge $(+5 \mathrm{~V})$ touches another piece. We placed a porous insulator between the different pieces, allowing a connection to be made only when users press down on the top layer (Fig 5). We sewed the pieces of silk onto a larger piece of non-conductive fabric using a conductive thread sewn on much like the copper tracks on a Printed Circuit Board (Fig 7). Each thread connects to a wire, which connects to a data acquisition board. The latest design uses the DataAcq DT 9800 Board [2] with 16 analog inputs, 8 digital inputs and a USB connector that allows the board to communicate with a computer. Visual $\mathrm{C}++$ code processes the signal from the board and detects which sensors are currently being pressed. Finally, the information from the $\mathrm{C}++$ program is sent to Macromedia Flash as input to the application that is being controlled. 


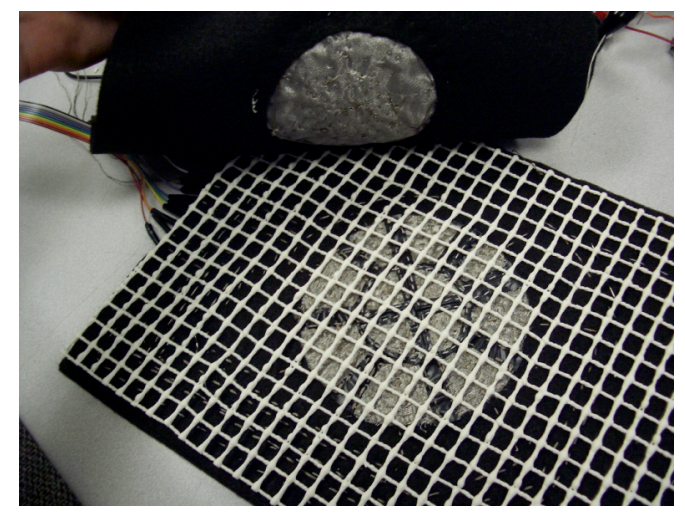

Fig 5. Image of the first prototype showing the different layers of the sensor: (top) circle of silk organza charged with $+5 \mathrm{~V}$, (middle) permeable insulator, and (bottom) smaller buttons made of silk organza.

\subsection{First Prototype}

As a proof of concept, we constructed our first working prototype using heavy felt as the non-conductive fabric. In addition, we used an anti-slip carpet pad made of rubber mesh as the insulator between the pieces of conductive fabric. The prototype functioned well; however, the thickness of the insulator made it quite noticeable when stroking the sunken ring. Also, stroking the felt created an uncomfortable amount of friction and caused the felt to ball up and fray a bit after use. Finally, this version of the fabric-circle-slider contained 8 soft-buttons around the ring, which caused it to lack sufficient granularity for rapid expressions from the user.

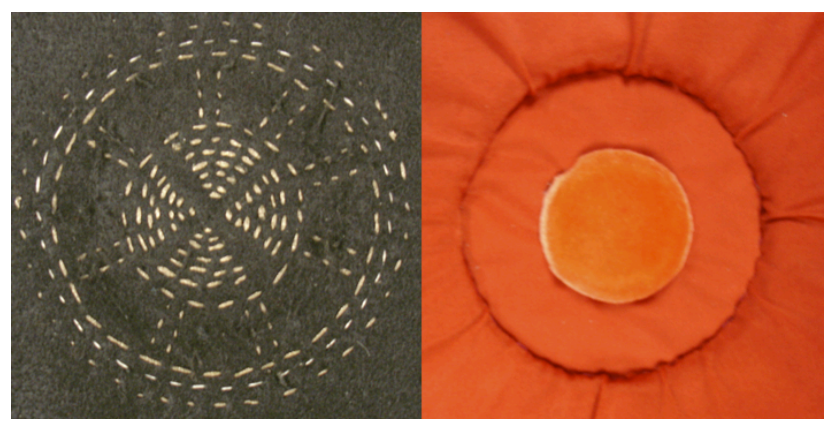


Fig 6. Top layers of the first prototype (left) and the second (right)

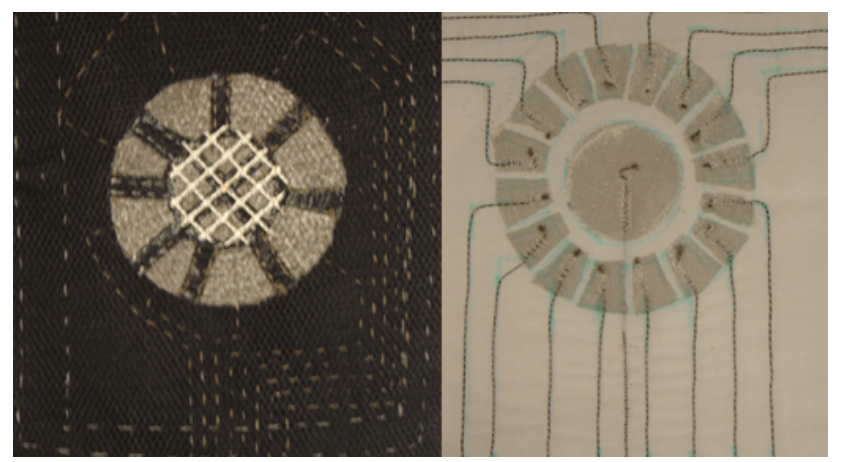

Fig 7. Button layout for the first (left) prototype and second (right)

\subsection{Prototype 2}

For our second prototype, we changed the feel of the device controller in several ways. First, we replaced the nonconductive felt with a silky and stretchy cotton knit. This made running a finger around the sunken ring a more pleasurable, tactile experience. Second, we dampened the feeling to the conductive threads that connected the soft-buttons to the computer with an addition layer of material between the threads and the top fabric users stroke. Third, we added a layer of rubber to the bottom on the widget, so it would not slide while being interacted with. Fourth, we replaced the bulky carpet pad with two layers of tulle, which worked well as a new porous insulator. Fifth, we added several layers of thin rubber on the inside to construct a smoother transition between the back of the armrest cover and outside edge of the sunken ring (Fig 8). 


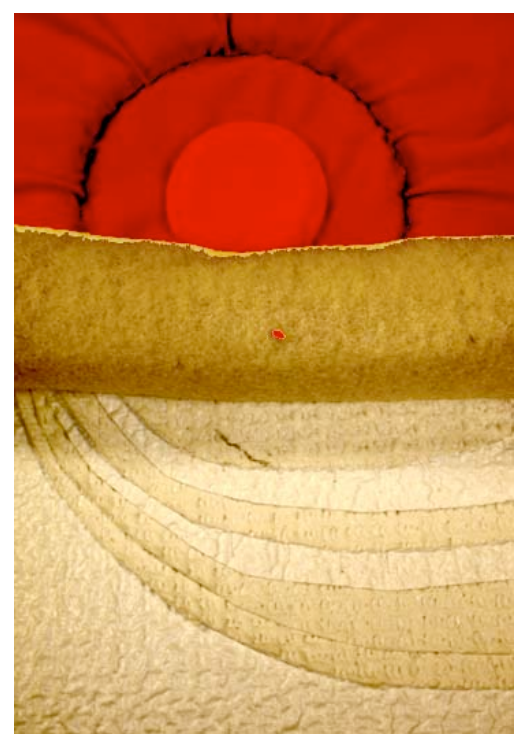

Fig 8. Layers of fabric used to create the outside edge of the sunken-ring.

In addition to changing the feel of the sensor, we also changed the hardware design. We increased the number of soft-buttons in the ring from 8 to 16 , increasing the sensitivity of the interaction expressions. We also changed the center of the ring by replacing the four soft-buttons originally arranged as a four-directional cursor with one large button made out of velvet, providing a tactile difference between the center button and the silky, sunken ring.

\section{Conclusion}

The increasing complexity of interaction in the home brought on by both the increasing number of devices related to as a single activity as well as the number of activities and tasks a single device supports has led to a decline in products that consider interaction aesthetics. Contextual integration-linking the interaction to the resources and elements found in the context-offers one approach for increasing the consideration of interaction aesthetics in product design. The fabric-ring-slider provides an early example of how designers can increase interaction aesthetics in this more complex space. From a "chair-ness" perspective, this widget integrates nicely into both the form and activities of the lounge chair. In addition, the softness of the widget captures some of the comfort and support provided by the chair. The generic nature of the input system and its support for three interaction expressions allows the widget to work with many different kinds of devices people want to control from their chair.

While a far from a being a completely representative example of a contextually integrated interaction design, the fabric-circle-slider does provide a pilot example for helping to explore this new design space. In the near future we plan a formal evaluation of the widget, comparing it to both traditional remote controls and to hard con- 
trols such as real buttons and knobs integrated into the chair. In addition, we plan to extend this specific interaction widget within the home, exploring how it might work as part of a throw pillow in the living room or a bedspread in the bedroom.

\section{References}

1. Crampton Smith, G.: The Hand that Rocks the Cradle. ID (May/June1995) 60-65.

2. Data Acquisition Hardware - DT 9800 Series:

http://www.datx.com/products_hardware/prod_dt9800-series.htm

3. Djajadiningrat, J.P., Gaver, W.W., Frens, J.W.: Interaction Relabelling and Extreme Characters: Methods for Exploring Aesthetic Interactions. Proceedings of DIS (2000) 66-71.

4. Forlizzi, J., DiSalvo, C., and Gemperle, F.: Assistive Robotics and an Ecology of Elders Living Independently in Their Homes. Journal of HCI Special Issue on Human-Robot Interaction, V19 N1/2 (2004) 25-59.

5. Forlizzi, J, DiSalvo, C., Zimmerman, J., Mutlu, B., Hurst, A.: The SenseChair: The Lounge Chair as an Intelligent Assistive Device for Elders. Proceedings of Designing for User Experience (2005).

6. Gaver, W., Bowers, J., Boucher. A., Gellerson, H., Pennington, S., Schmidt, A., Steed, A., Villars, N., Walker, B.: The Drift Table: Designing for Ludic Engagement, Proceedings of CHI (2004) 885-900.

7. Hurst, A., Zimmerman, J., Atkeson, C., Forlizzi, J.: The Sense Lounger: Establishing an Ubicomp Beachhead in Elders' Homes. Proceeding of CHI (2005) 1467-1470.

8. Ishii, H., Ullmer, B.: Tangible Bits: Towards seamless Interfaces Between People, Bits, and Atoms. Proceedings of CHI (1997) 234-241.

9. Ishii, H., Mazalek, A., Lee, J.: Bottles as a Minimal Interface to Access Digital Information. Proceedings of CHI (2001) 187-188.

10. Project on People and Robots: http://www.peopleandrobots.org/

11. Wensveen, S., Overbeeke, C.J., and Djajadiningrat, T.: Touch Me, Hit Me, and I Know How You Feel: a Design Approach to Emotionally Rich Interaction. Proceedings of DIS (2000) 48-52.

12. Wensveen, S.A.G., Djajadiningrat, J.P., Overbeeke, C.J.: Interaction Frogger: A Design Framework to Couple Action and Function Through Feedback and Feedforward. Proceedings of DIS (2004) 177-184.

13. Zimmerman, J., Ayoob, E., Forlizzi, J.: Searching for Intrinsic Value in Interaction: Reflections on the Conceptual Designs of Digital Music Players. Proceedings of DPPI (2005) 